\title{
Weingarten spacelike hypersurfaces in a de Sitter space
}

\author{
Junfeng Chen and Shichang Shu
}

\begin{abstract}
We study some Weingarten spacelike hypersurfaces in a de Sitter space $S_{1}^{n+1}(1)$. If the Weingarten spacelike hypersurfaces have two distinct principal curvatures, we obtain two classification theorems which give some characterization of the Riemannian product $H^{k}\left(1-\operatorname{coth}^{2} \varrho\right) \times$ $S^{n-k}\left(1-\tanh ^{2} \varrho\right), 1<k<n-1$ in $S_{1}^{n+1}(1)$, the hyperbolic cylinder $H^{1}\left(1-\operatorname{coth}^{2} \varrho\right) \times S^{n-1}\left(1-\tanh ^{2} \varrho\right)$ or spherical cylinder $H^{n-1}(1-$ $\left.\operatorname{coth}^{2} \varrho\right) \times S^{1}\left(1-\tanh ^{2} \varrho\right)$ in $S_{1}^{n+1}(1)$.
\end{abstract}

\section{Introduction}

By an $(n+1)$-dimensional Lorentzian space form $M_{1}^{n+1}(c)$ we mean a de Sitter space $S_{1}^{n+1}(c)$, a Minkowski space $R_{1}^{n+1}$ or an anti-de Sitter space $H_{1}^{n+1}(c)$, according to $c>0, c=0$ or $c<0$, respectively. That is, a Lorentzian space form $M_{1}^{n+1}(c)$ is a complete simply connected $(n+1)$-dimensional Lorentzian manifold with constant curvature $c$. A hypersurface in a Lorentzian manifold is said to be spacelike if the induced metric on the hypersurface is positive definite.

We know that hypersurfaces with prescribed curvature are called Weingarten hypersurfaces. Weingarten hypersurfaces have been studied by many authors from the point of view of geometric analysis (see $[6,10])$. In this article, we introduce some special Weingarten spacelike hypersurfaces in an

Key Words: Weingarten spacelike hypersurface, scalar curvature, mean curvature, squared norm of second fundamental form.

2010 Mathematics Subject Classification: Primary 53C42; Secondary 53A10.

Received: January, 2011.

Revised: January, 2012.

Accepted: February, 2012. 
$(n+1)$-dimensional de Sitter space $S_{1}^{n+1}(1)$ and study the curvatures and geometric properties by moving frame method. We give the definition: A spacelike hypersurface in a de Sitter space $S_{1}^{n+1}(1)$ is called a linear Weingarten spacelike hypersurface if the scalar curvature $R$ and the mean curvature $H$ satisfy the linear relation $\alpha R+\beta H+\gamma=0$, where $\alpha, \beta$ and $\gamma$ are constants such that $\alpha^{2}+\beta^{2} \neq 0$. We easily see that if $\alpha=0, \beta \neq 0$, a linear Weingarten spacelike hypersurface reduces to a spacelike hypersurface with constant mean curvature; if $\beta=0, \alpha \neq 0$, it reduces to a spacelike hypersurface with constant scalar curvature; if $\gamma=0, \alpha \neq 0, \beta \neq 0$, it reduces to a spacelike hypersurface with the scalar curvature and the mean curvature being linearly related.

We notice that the linear Weingarten spacelike hypersurface is a natural generalization of spacelike hypersurface with constant mean curvature or with constant scalar curvature or the scalar curvature and the mean curvature being linearly related, and the latter three kinds of spacelike hypersurfaces have been intensively studied by many authors (see $[2,5,7,8,12]$ ). On the other hand, we also notice that Hou and Yang [9] recently introduce the so called linear Weingarten spacelike hypersurfaces, that is, spacelike hypersurfaces satisfying $r=a H+b$, where $r=\frac{1}{n(n-1) R}$ is the normalized scalar curvature and $H$ the mean curvature. They give a classification of such hypersurfaces by the sectional curvature or the length of the second fundamental form. We note that Hou and Yang's definition include spacelike hypersurfaces with constant scalar curvature or with the scalar curvature and the mean curvature being linearly related, but not include spacelike hypersurfaces with constant mean curvature. Thus, we see that the definition of this article is a generalization of Hou and Yang's in [9]. For the investigation of the so called Ruled Weingarten hypersurfaces and the low dimensional linear Weingarten surfaces, one can see [4] and [3].

We denote by $\left(h_{i j}\right)$ the second fundamental form of spacelike hypersurface in a de Sitter space $S_{1}^{n+1}(1)$, by $H=\frac{1}{n} \sum_{i=1}^{n} h_{i i}$ the mean curvature, and by $\phi_{i j}$ the tensor $h_{i j}-H \delta_{i j}$ of the trace free part of the second fundamental form $h_{i j}$. Let $S$ and $\rho^{2}$ be the square of the length of $\left(h_{i j}\right)$ and $\left(\phi_{i j}\right)$, respectively. We easily know that $\rho^{2}=S-n H^{2}$ and $\rho^{2}=0$ if and only if the spacelike hypersurface is umbilical. We notice that $\rho^{2}$ and $H$ are two important invariants of the spacelike hypersurface. It is natural for us to consider spacelike hypersurface with non-zero constant $\rho^{2}$, we call it a constant $\rho$ Weingarten spacelike hypersurface. Obviously, a constant $\rho$ Weingarten spacelike hypersurface is not umbilical.

From now on, we consider the linear Weingarten spacelike hypersurfaces and constant $\rho$ Weingarten spacelike hypersurfaces in a de Sitter space $S_{1}^{n+1}(1)$ with two distinct principal curvatures. Since the spacelike hypersurfaces with constant mean curvature are linear Weingarten spacelike hypersurfaces, we in- 
troduce the well-known standard models of complete linear Weingarten spacelike hypersurfaces and the constant $\rho$ Weingarten spacelike hypersurfaces in a de Sitter space $S_{1}^{n+1}(1)$ :

Let $T_{k, \varrho}:=\left\{x \in S_{1}^{n+1}(1) \mid-x_{0}^{2}+x_{1}^{2}+\cdots+x_{k}^{2}=-\sinh ^{2} \varrho\right\}, \varrho>0,1 \leq k \leq$ $n-1$. Then $T_{k, \varrho}$ has two distinct constant principal curvatures

$$
\lambda_{1}=\cdots=\lambda_{k}=\operatorname{coth} \varrho, \quad \lambda_{k+1}=\cdots=\lambda_{n}=\tanh \varrho .
$$

Moreover, $T_{k, \varrho}$ is isometric to the Riemannian product $H^{k}\left(1-\operatorname{coth}^{2} \varrho\right) \times$ $S^{n-k}\left(1-\tanh ^{2} \varrho\right) \hookrightarrow S_{1}^{n+1}(1)$. In particular, the Riemannin product $H^{1}(1-$ $\left.\operatorname{coth}^{2} \varrho\right) \times S^{n-1}\left(1-\tanh ^{2} \varrho\right)$ or $H^{n-1}\left(1-\operatorname{coth}^{2} \varrho\right) \times S^{1}\left(1-\tanh ^{2} \varrho\right)$ is called a hyperbolic cylinder or a spherical cylinder in $S_{1}^{n+1}(1)$. By a direct calculation, we have $\rho^{2}=\frac{n-1}{n}(\operatorname{coth} \varrho-\tanh \varrho)^{2}$ and the mean curvature

$$
H=\frac{(n-2) \sigma}{2 n} \sqrt{\frac{n}{n-1}} \rho-\sqrt{\frac{n \rho^{2}}{4(n-1)}+1},
$$

or

$$
H=\frac{(n-2) \sigma}{2 n} \sqrt{\frac{n}{n-1}} \rho+\sqrt{\frac{n \rho^{2}}{4(n-1)}+1},
$$

where $\sigma= \pm 1$ is the sign of the difference $\operatorname{coth} \varrho-\tanh \varrho$.

We shall prove the following:

Theorem 1.1. Let $M^{n}$ be an $n(n \geq 3)$-dimensional complete connected and oriented linear Weingarten spacelike hypersurface or constant $\rho$ Weingarten spacelike hypersurface in a de Sitter space $S_{1}^{n+1}(1)$ with two distinct principal curvatures. Then

(1) if the multiplicities of both principal curvatures are greater than 1, then $M^{n}$ is isometric to the Riemannian product $H^{k}\left(1-\operatorname{coth}^{2} \varrho\right) \times S^{n-k}(1-$ $\left.\tanh ^{2} \varrho\right), 1<k<n-1$.

(2) if $M^{n}$ has two distinct principal curvatures $\lambda$ and $\mu$ of multiplicities $n-1$ and 1 and the sectional curvature of $M^{n}$ is nonnegative, then

(i) for linear Weingarten spacelike hypersurface, if $\lambda \neq \frac{\beta}{2 \alpha n(n-1)}, \gamma \alpha+$ $\alpha^{2} n(n-1)=-\frac{\beta^{2}}{4 n(n-1)}$ and $\frac{2 n^{4}(n-1)}{n-2}<\frac{\beta^{2}}{\alpha^{2}}<4 n^{2}(n-1)^{2}$, then $M^{n}$ is isometric to the hyperbolic cylinder $H^{1}\left(1-\operatorname{coth}^{2} \varrho\right) \times S^{n-1}\left(1-\tanh ^{2} \varrho\right)$ or spherical cylinder $H^{n-1}\left(1-\operatorname{coth}^{2} \varrho\right) \times S^{1}\left(1-\tanh ^{2} \varrho\right)$;

(ii) for constant $\rho$ Weingarten spacelike hypersurface, if $\lambda>0$, then $M^{n}$ is isometric to the hyperbolic cylinder $H^{1}\left(1-\operatorname{coth}^{2} \varrho\right) \times S^{n-1}\left(1-\tanh ^{2} \varrho\right)$ or spherical cylinder $H^{n-1}\left(1-\operatorname{coth}^{2} \varrho\right) \times S^{1}\left(1-\tanh ^{2} \varrho\right)$. 
Denote by $P(t)$ and $S(t)$ the following functions:

$$
P(t)=1-\frac{\beta}{4 \alpha(n-1)} t+\frac{n-2}{2} t^{2},
$$

and

$$
S(t)=\frac{n^{2}}{4} t^{2}-\frac{(n-2) \beta}{4 \alpha(n-1)} t+\frac{\beta^{2}}{16 \alpha^{2}(n-1)^{2}} .
$$

From Lemma 3.3, we know that $P(t)$ has two distinct real roots $t_{1}, t_{2}$. On the other hand, denote by $\tilde{P}(t)$ and $H(t)$ the following functions:

$$
\tilde{P}(t)=-t^{2}+\sigma \sqrt{\frac{n}{n-1}} \rho t+1,
$$

and

$$
H(t)=t-\frac{\sigma}{n} \sqrt{\frac{n}{n-1}} \rho .
$$

We know that $\tilde{P}(t)$ has two distinct real roots $\tilde{t_{1}}, \tilde{t_{2}}$, namely,

$$
\begin{aligned}
& \tilde{t_{1}}=-\frac{\sigma}{2} \sqrt{\frac{n}{n-1}} \rho-\sqrt{\frac{n \rho^{2}}{4(n-1)}+1}, \\
& \tilde{t_{2}}=-\frac{\sigma}{2} \sqrt{\frac{n}{n-1}} \rho+\sqrt{\frac{n \rho^{2}}{4(n-1)}+1},
\end{aligned}
$$

We can prove the following:

Theorem 1.2. $\quad$ Let $M^{n}$ be an $n(n \geq 3)$-dimensional complete connected and oriented linear Weingarten spacelike hypersurface or constant $\rho$ Weingarten spacelike hypersurface in a de Sitter space $S_{1}^{n+1}(1)$ with two distinct principal curvatures $\lambda$ and $\mu$ of multiplicities $n-1$ and 1 . Then

(1) for linear Weingarten spacelike hypersurface, if $\lambda \neq \frac{\beta}{2 \alpha n(n-1)}, \gamma \alpha+$ $\alpha^{2} n(n-1)=-\frac{\beta^{2}}{4 n(n-1)}, \frac{2 n^{4}(n-1)}{n-2}<\frac{\beta^{2}}{\alpha^{2}}<4 n^{2}(n-1)^{2}$ and the squared norm of the second fundamental form of $M^{n}$ satisfies one of the following conditions

(i) $\min \left(S\left(t_{1}\right), S\left(t_{2}\right)\right) \leq S \leq \max \left(S\left(t_{1}\right), S\left(t_{2}\right)\right)$, or

(ii) $S \geq \max \left(S\left(t_{1}\right), S\left(t_{2}\right)\right)$, or

(iii) $S \leq \min \left(S\left(t_{1}\right), S\left(t_{2}\right)\right)$, then $M^{n}$ is isometric to the hyperbolic cylinder $H^{1}\left(1-\operatorname{coth}^{2} \varrho\right) \times S^{n-1}\left(1-\tanh ^{2} \varrho\right)$ or spherical cylinder $H^{n-1}\left(1-\operatorname{coth}^{2} \varrho\right) \times$ $S^{1}\left(1-\tanh ^{2} \varrho\right)$, where $t_{1}, t_{2}$ are the two distinct real roots of (1.1) and $S(t)$ is denoted by (1.2); 
(2) for constant $\rho$ Weingarten spacelike hypersurface, if the mean curvature of $M^{n}$ satisfies one of the following conditions

(i) $H \geq H\left(\tilde{t_{2}}\right)$, or

(ii) $H \leq H\left(\tilde{t_{1}}\right)$, or

(iii) if $H\left(\tilde{t_{1}}\right) \leq H \leq H\left(\tilde{t_{2}}\right)$, then $M^{n}$ is isometric to the hyperbolic cylinder $H^{1}\left(1-\operatorname{coth}^{2} \varrho\right) \times S^{n-1}\left(1-\tanh ^{2} \varrho\right)$ or spherical cylinder $H^{n-1}\left(1-\operatorname{coth}^{2} \varrho\right) \times$ $S^{1}\left(1-\tanh ^{2} \varrho\right)$, where $\tilde{t_{1}}, \tilde{t_{2}}$ are the two distinct real roots of (1.3) and $H(t)$ is denoted by (1.4).

\section{Preliminaries}

Let $M^{n}$ be an $n$-dimensional space-like hypersurface in an $(\mathrm{n}+1)$-dimensional de Sitter space $S_{1}^{n+1}(c)$ with constant sectional curvature $c(c>0)$. We choose a local field of semi-Riemannian orthonormal frames $\left\{e_{1}, \cdots, e_{n+1}\right\}$ in $S_{1}^{n+1}(c)$ such that at each point of $M^{n},\left\{e_{1}, \cdots, e_{n}\right\}$ span the tangent space of $M^{n}$ and form an orthonormal frame there. We use the following convention on the range of indices:

$$
1 \leq A, B, C, \cdots \leq n+1 ; \quad 1 \leq i, j, k, \cdots \leq n .
$$

Let $\left\{\omega_{1}, \cdots, \omega_{n+1}\right\}$ be the dual frame field so that the semi-Riemannian metric of $S_{1}^{n+1}(c)$ is given by $d \bar{s}^{2}=\sum_{i} \omega_{i}^{2}-\omega_{n+1}^{2}=\sum_{A} \epsilon_{A} \omega_{A}^{2}$, where $\epsilon_{i}=1$ and $\epsilon_{n+1}=-1$.

The structure equations of $S_{1}^{n+1}(c)$ are given by

$$
\begin{gathered}
d \omega_{A}=\sum_{B} \epsilon_{B} \omega_{A B} \wedge \omega_{B}, \quad \omega_{A B}+\omega_{B A}=0, \\
d \omega_{A B}=\sum_{C} \epsilon_{C} \omega_{A C} \wedge \omega_{C B}+\Omega_{A B},
\end{gathered}
$$

where

$$
\begin{aligned}
\Omega_{A B} & =-\frac{1}{2} \sum_{C, D} K_{A B C D} \omega_{C} \wedge \omega_{D}, \\
K_{A B C D} & =\epsilon_{A} \epsilon_{B} c\left(\delta_{A C} \delta_{B D}-\delta_{A D} \delta_{B C}\right) .
\end{aligned}
$$

Restrict these forms to $M^{n}$, we have

$$
\omega_{n+1}=0 .
$$

Cartan's Lemma implies that

$$
\omega_{n+1 i}=\sum_{j} h_{i j} \omega_{j}, \quad h_{i j}=h_{j i} .
$$


The structure equations of $M^{n}$ are

$$
\begin{gathered}
d \omega_{i}=\sum_{j} \omega_{i j} \wedge \omega_{j}, \quad \omega_{i j}+\omega_{j i}=0, \\
d \omega_{i j}=\sum_{k} \omega_{i k} \wedge \omega_{k j}-\frac{1}{2} \sum_{k, l} R_{i j k l} \omega_{k} \wedge \omega_{l}, \\
R_{i j k l}=c\left(\delta_{i k} \delta_{j l}-\delta_{i l} \delta_{j k}\right)-\left(h_{i k} h_{j l}-h_{i l} h_{j k}\right),
\end{gathered}
$$

where $R_{i j k l}$ are the components of the curvature tensor of $M^{n}$ and

$$
h=\sum_{i, j} h_{i j} \omega_{i} \otimes \omega_{j}
$$

is the second fundamental form of $M^{n}$.

From the above equation, we have

$$
R-n(n-1) c=S-n^{2} H^{2},
$$

where $R$ is the scalar curvature of $M^{n}, H$ is the mean curvature, and $S=\sum_{i, j} h_{i j}^{2}$ is the squared norm of the second fundamental form of $M^{n}$.

We choose $e_{1}, \cdots, e_{n}$ such that $h_{i j}=\lambda_{i} \delta_{i j}$. From (2.6) we have

$$
\omega_{n+1 i}=\lambda_{i} \omega_{i}, \quad i=1,2, \cdots, n .
$$

From the curvature forms of $S_{1}^{n+1}(c)$,

$$
\begin{aligned}
\Omega_{n i} & =-\frac{1}{2} \sum_{C, D} K_{n i C D} \omega_{C} \wedge \omega_{D} \\
& =\frac{1}{2} \sum_{C, D} c\left(\delta_{n C} \delta_{i D}-\delta_{n D} \delta_{i C}\right) \omega_{C} \wedge \omega_{D} \\
& =c \omega_{n} \wedge \omega_{i} .
\end{aligned}
$$

Since the covariant derivative of the second fundamental form $h_{i j}$ of $M^{n}$ is defined by

$$
\sum_{k} h_{i j k} \omega_{k}=d h_{i j}+\sum_{k} h_{i k} \omega_{k j}+\sum_{k} h_{k j} \omega_{k i}
$$

we have

$$
\sum_{k} h_{i j k} \omega_{k}=\delta_{j i} d \lambda_{j}+\left(\lambda_{i}-\lambda_{j}\right) \omega_{i j}
$$

Putting $\psi_{i j}=\left(\lambda_{i}-\lambda_{j}\right) \omega_{i j}$, we have $\psi_{i j}=\psi_{j i}$ and

$$
\psi_{i j}+\delta_{i j} d \lambda_{j}=\sum_{k} h_{i j k} \omega_{k}
$$

where $h_{i j k}$ satisfy

$$
h_{i j k}=h_{j i k}=h_{i k j} .
$$




\section{Proof of Theorems}

We firstly state the following Proposition 3.1 original due to Otsuki [11] for Riemannian space forms.

Proposition 3.1. Let $M^{n}$ be a spacelike hypersurface in an $(n+1)$-dimensional de Sitter space $S_{1}^{n+1}(c)$ such that the multiplicities of the principal curvatures are constant. Then the distribution of the space of the principal vectors corresponding to each principal curvature is completely integrable. In particular, if the multiplicity of a principal curvature is greater than 1, then this principal curvature is constant on each integral submanifold of the corresponding distribution of the space of the principal vectors.

Proof of (1) in Theorem 1.1. Let $\lambda, \mu$ be the principal curvatures of multiplicities $k$ and $n-k$ respectively, where $1<k<n-1$. If $M^{n}$ is a linear Weingarten spacelike hypersurface in $S_{1}^{n+1}(1)$, by (2.11) and $\alpha R+\beta H+\gamma=0$, we have

$$
\alpha S+\beta H-\alpha n^{2} H^{2}+\alpha n(n-1)+\gamma=0,
$$

if $M^{n}$ is a constant $\rho$ Weingarten spacelike hypersurface, we have

$$
S-n H^{2}=\rho^{2}=\text { constant }
$$

Substituting $n H=k \lambda+(n-k) \mu$ and $S=k \lambda^{2}+(n-k) \mu^{2}$ in (3.1) or (3.2), we obtain an equation of $\lambda$ and $\mu$, denoted by

$$
\mathcal{F}(\lambda, \mu)=0 .
$$

Denote by $\mathcal{D}_{\lambda}$ and $\mathcal{D}_{\mu}$ the integral submanifolds of the corresponding distribution of the space of principal vectors corresponding to the principal curvature $\lambda$ and $\mu$, respectively. From Proposition 3.1, we know that $\lambda$ is constant on $\mathcal{D}_{\lambda}$. From (3.3), we infer that $\mu$ is constant on $\mathcal{D}_{\lambda}$. By making use of Proposition 3.1 again, we have $\mu$ is constant on $\mathcal{D}_{\mu}$. Therefore, we know that $\mu$ is constant on $M^{n}$. By the same assertion we know that $\lambda$ is constant on $M^{n}$. Therefore $M^{n}$ is isoparametric. By the congruence Theorem of Abe, Koike and Yamaguchi (see Theorem 5.1 of [1]), we know that $M^{n}$ is isometric to the Riemannian product $H^{k}\left(1-\operatorname{coth}^{2} \varrho\right) \times S^{n-k}\left(1-\tanh ^{2} \varrho\right)$ and $1<k<n-1$. This completes the proof of (1) in Theorem 1.1.

Remark. In fact, we notice that the result of (1) in Theorem 1.1 is true for any Weingarten spacelike hypersurfaces satisfying a differentiable function relating the scalar curvature, the mean curvature and the squared norm of the second fundamental form of $M^{n}$. 
Let $M^{n}$ be an $n$-dimensional complete linear Weingarten spaclike hypersurface or constant $\rho$ Weingarten spacelike hypersurface with two distinct principal curvatures one of which is simple, that is, without loss of generality, we may assume

$$
\lambda_{1}=\lambda_{2}=\cdots=\lambda_{n-1}=\lambda, \quad \lambda_{n}=\mu,
$$

where $\lambda_{i}$ for $i=1,2, \cdots, n$ are the principal curvatures of $M^{n}$.

If $M^{n}$ is a linear Weingarten spacelike hypersurface, since we assume that $n(n-1) \alpha^{2}+\gamma \alpha=-\frac{\beta^{2}}{4 n(n-1)}$, from $(2.11)$ and $\alpha R+\beta H+\gamma=0$, we obtain that for $c=1$

$$
\begin{aligned}
\left(\frac{\beta}{n}-2 \alpha(n-1) \lambda\right) \mu & -\alpha(n-1)(n-2) \lambda^{2} \\
& +\frac{n-1}{n} \beta \lambda-\frac{\beta^{2}}{4 n(n-1) \alpha}=0 .
\end{aligned}
$$

Since $\lambda \neq \frac{\beta}{2 \alpha n(n-1)}$, from (3.4), we have

$$
\begin{gathered}
\mu=-\frac{2 \alpha(n-1)(n-2) \lambda-\beta}{4 \alpha(n-1)}, \\
d \mu=-\frac{n-2}{2} d \lambda .
\end{gathered}
$$

and

$$
\lambda-\mu=n \frac{2 \alpha n(n-1) \lambda-\beta}{4 \alpha n(n-1)} .
$$

If $M^{n}$ is a constant $\rho$ Weingarten spacelike hypersurface, from $\rho^{2}=S-n H^{2}$, we obtain that

$$
\frac{n-1}{n}(\lambda-\mu)^{2}=\rho^{2}
$$

Thus, we have

$$
\lambda-\mu=\sigma \sqrt{\frac{n}{n-1}} \rho \neq 0,
$$

where $\sigma= \pm 1$ is the sign of the difference $\lambda-\mu$. Therefore, we know that

$$
\begin{gathered}
\mu=\lambda-\sigma \sqrt{\frac{n}{n-1}} \rho, \\
d \mu=d \lambda .
\end{gathered}
$$

Let $\varpi=|2 \alpha n(n-1) \lambda-\beta|^{-\frac{2}{n}}$ for linear Weingarten spacelike hypersurface and $\varpi=e^{-\left(\lambda / \sigma \sqrt{\frac{n}{n-1}} \rho\right)}$ for constant $\rho$ Weingarten spacelike hypersurface. 
We denote the integral submanifold through $x \in M^{n}$ corresponding to $\lambda$ by $M_{1}^{n-1}(x)$. Putting

$$
d \lambda=\sum_{k=1}^{n} \lambda,_{k} \omega_{k}, \quad d \mu=\sum_{k=1}^{n} \mu, \omega_{k} \omega_{k}
$$

From Proposition 3.1, we have

$$
\lambda,_{1}=\lambda,_{2}=\cdots=\lambda,_{n-1}=0 \text { on } M_{1}^{n-1}(x) .
$$

From (3.6) or (3.11), we have

$$
\mu,,_{1}=\mu,_{2}=\cdots=\mu,_{n-1}=0 \quad \text { on } M_{1}^{n-1}(x) .
$$

In this case, we may consider locally $\lambda$ is a function of the arc length $s$ of the integral curve of the principal vector field $e_{n}$ corresponding to the principal curvature $\mu$. From (2.14) and (3.13), we have for $1 \leq j \leq n-1$,

$$
\begin{aligned}
d \lambda & =d \lambda_{j}=\sum_{k=1}^{n} h_{j j k} \omega_{k} \\
& =\sum_{k=1}^{n-1} h_{j j k} \omega_{k}+h_{j j n} \omega_{n}=\lambda,_{n} \omega_{n} .
\end{aligned}
$$

Therefore, we have

$$
h_{j j k}=0, \quad 1 \leq k \leq n-1, \quad \text { and } \quad h_{j j n}=\lambda,{ }_{n} .
$$

By (2.14) and (3.14), we have

$$
\begin{aligned}
d \mu & =d \lambda_{n}=\sum_{k=1}^{n} h_{n n k} \omega_{k} \\
& =\sum_{k=1}^{n-1} h_{n n k} \omega_{k}+h_{n n n} \omega_{n}=\sum_{i=1}^{n} \mu,_{i} \omega_{i}=\mu,_{n} \omega_{n} .
\end{aligned}
$$

Thus, we obtain

$$
h_{n n k}=0, \quad 1 \leq k \leq n-1, \quad \text { and } \quad h_{n n n}=\mu,_{n} .
$$

From (3.6) or (3.11), we get

$$
h_{n n n}=\mu,_{n}=-\frac{n-2}{2} \lambda,_{n},
$$


or

$$
h_{n n n}=\mu,_{n}=\lambda,_{n} .
$$

From the definition of $\psi_{i j}$, if $i \neq j$, we have $\psi_{i j}=0$ for $1 \leq i \leq n-1$ and $1 \leq j \leq n-1$. Therefore, from (2.14), if $i \neq j$ and $1 \leq i \leq n-1$ and $1 \leq j \leq n-1$ we have

$$
h_{i j k}=0, \text { for any } k \text {. }
$$

By (2.14), (3.16), (3.18), (3.19) or (3.20) and (3.21), we get

$$
\psi_{j n}=\sum_{k=1}^{n} h_{j n k} \omega_{k}=h_{j j n} \omega_{j}+h_{j n n} \omega_{n}=\lambda,_{n} \omega_{j}
$$

From the definition of $\psi_{i j},(3.7)$ or (3.9) and (3.22) we have

$$
\omega_{j n}=\frac{\psi_{j n}}{\lambda-\mu}=\frac{\lambda,_{n}}{\lambda-\mu} \omega_{j}=\frac{4 \alpha n(n-1) \lambda,,_{n}}{n[2 \alpha n(n-1) \lambda-\beta]} \omega_{j},
$$

or

$$
\omega_{j n}=\frac{\lambda, n}{\lambda-\mu} \omega_{j}=\frac{\lambda,,_{n}}{\sigma \sqrt{\frac{n}{n-1}} \rho} \omega_{j} .
$$

Thus, from the structure equations of $M^{n}$ we have

$$
d \omega_{n}=\sum_{k=1}^{n-1} \omega_{k} \wedge \omega_{k n}+\omega_{n n} \wedge \omega_{n}=0
$$

Therefore, we may put $\omega_{n}=d s$. By (3.15) and (3.17), we get

$$
d \lambda=\lambda,_{n} d s, \quad \lambda_{n}=\frac{d \lambda}{d s}
$$

and

$$
d \mu=\mu,_{n} d s, \quad \mu,_{n}=\frac{d \mu}{d s} .
$$

From (3.23) or (3.24), we have

$$
\omega_{j n}=\frac{4 \alpha n(n-1) \frac{d \lambda}{d s}}{n[2 \alpha n(n-1) \lambda+\beta]} \omega_{j}=\frac{d\left\{\log |2 \alpha n(n-1) \lambda-\beta|^{\frac{2}{n}}\right\}}{d s} \omega_{j},
$$

or

$$
\omega_{j n}=\frac{\frac{d \lambda}{d s}}{\sigma \sqrt{\frac{n}{n-1}} \rho} \omega_{j}=\frac{d\left(\lambda / \sigma \sqrt{\frac{n}{n-1}} \rho\right)}{d s} \omega_{j} .
$$


From (3.25) or (3.26), (2.13) and the structure equations of $S_{1}^{n+1}(c)$, we have

$$
\begin{aligned}
d \omega_{j n} & =\sum_{k=1}^{n-1} \omega_{j k} \wedge \omega_{k n}+\omega_{j n} \wedge \omega_{n n}+\omega_{j n+1} \wedge \omega_{n+1 n}+\Omega_{j n} \\
& =\sum_{k=1}^{n-1} \omega_{j k} \wedge \omega_{k n}+\omega_{j n+1} \wedge \omega_{n+1 n}-c \omega_{j} \wedge \omega_{n} \\
& =\frac{d\left\{\log |2 \alpha n(n-1) \lambda-\beta|^{\frac{2}{n}}\right\}}{d s} \sum_{k=1}^{n-1} \omega_{j k} \wedge \omega_{k}-(c-\lambda \mu) \omega_{j} \wedge d s
\end{aligned}
$$

or

$$
d \omega_{j n}=\frac{d\left(\lambda / \sigma \sqrt{\frac{n}{n-1}} \rho\right)}{d s} \sum_{k=1}^{n-1} \omega_{j k} \wedge \omega_{k}-(c-\lambda \mu) \omega_{j} \wedge d s .
$$

From (3.25) or (3.26), we have

$$
\begin{aligned}
d \omega_{j n}= & \frac{d^{2}\left\{\log |2 \alpha n(n-1) \lambda-\beta|^{\frac{2}{n}}\right\}}{d s^{2}} d s \wedge \omega_{j}+\frac{d\left\{\log |2 \alpha n(n-1) \lambda-\beta|^{\frac{2}{n}}\right\}}{d s} d \omega_{j} \\
= & \frac{d^{2}\left\{\log |2 \alpha n(n-1) \lambda-\beta|^{\frac{2}{n}}\right\}}{d s^{2}} d s \wedge \omega_{j}+\frac{d\left\{\log |2 \alpha n(n-1) \lambda-\beta|^{\frac{2}{n}}\right\}}{d s} \sum_{k=1}^{n} \omega_{j k} \wedge \omega_{k} \\
= & \left\{-\frac{d^{2}\left\{\log |2 \alpha n(n-1) \lambda-\beta|^{\frac{2}{n}}\right\}}{d s^{2}}+\left[\frac{d\left\{\log |2 \alpha n(n-1) \lambda-\beta|^{\frac{2}{n}}\right\}}{d s}\right]^{2}\right\} \omega_{j} \wedge d s \\
& +\frac{d\left\{\log |2 \alpha n(n-1) \lambda-\beta|^{\frac{2}{n}}\right\}}{d s} \sum_{k=1}^{n-1} \omega_{j k} \wedge \omega_{k} .
\end{aligned}
$$

or

$$
\begin{aligned}
d \omega_{j n}=\{ & \left.-\frac{d^{2}\left(\lambda / \sigma \sqrt{\frac{n}{n-1}} \rho\right)}{d s^{2}}+\left[\frac{d\left(\lambda / \sigma \sqrt{\frac{n}{n-1}} \rho\right)}{d s}\right]^{2}\right\} \omega_{j} \wedge d s \\
& +\frac{d\left(\lambda / \sigma \sqrt{\frac{n}{n-1}} \rho\right)}{d s} \sum_{k=1}^{n-1} \omega_{j k} \wedge \omega_{k} .
\end{aligned}
$$

From (3.27) and (3.29) or (3.28) and (3.30), we have

$$
\begin{aligned}
& \frac{d^{2}\left\{\log |2 \alpha n(n-1) \lambda-\beta|^{\frac{2}{n}}\right\}}{d s^{2}} \\
& -\left\{\frac{d\left\{\log |2 \alpha n(n-1) \lambda-\beta|^{\frac{2}{n}}\right\}}{d s}\right\}^{2}-(c-\lambda \mu)=0 .
\end{aligned}
$$


or

$$
\frac{d^{2}\left(\lambda / \sigma \sqrt{\frac{n}{n-1}} \rho\right)}{d s^{2}}-\left\{\frac{d\left(\lambda / \sigma \sqrt{\frac{n}{n-1}} \rho\right)}{d s}\right\}^{2}-(c-\lambda \mu)=0 .
$$

Since we define $\varpi=|2 \alpha n(n-1) \lambda-\beta|^{-\frac{2}{n}}$ for linear Weingarten spacelike hypersurface and $\varpi=e^{-\left(\lambda / \sigma \sqrt{\frac{n}{n-1}} \rho\right)}$ for constant $\rho$ Weingarten spacelike hypersurface, from $(3.31)$ or (3.32), we obtain

$$
\frac{d^{2} \varpi}{d s^{2}}+\varpi(c-\lambda \mu)=0
$$

We can prove the following Lemma:

Lemma 3.2. (1) If $1-\frac{\beta^{2}}{4 \alpha^{2} n^{2}(n-1)^{2}}>0$, then the positive function $\varpi=$ $|2 \alpha n(n-1) \lambda-\beta|^{-\frac{2}{n}}$ is bounded.

(2) If $\lambda>0$, then the positive function $\varpi=e^{-\left(\lambda / \sigma \sqrt{\frac{n}{n-1}} \rho\right)}$ is bounded.

Proof. (1) Let $c=1$. From (3.5) and (3.33), we get

$$
\frac{d^{2} \varpi}{d s^{2}}+\varpi\left(1-\frac{\beta}{4 \alpha(n-1)} \lambda+\frac{n-2}{2} \lambda^{2}\right)=0 .
$$

Since $\varpi=|2 \alpha n(n-1) \lambda-\beta|^{-\frac{2}{n}}$, we have

$$
\lambda=\frac{ \pm \varpi^{-\frac{n}{2}}+\beta}{2 \alpha n(n-1)} \text {. }
$$

Thus, we have from (3.34) that

$$
\frac{d^{2} \varpi}{d s^{2}}+\varpi\left(1-\frac{\beta\left( \pm \varpi^{-\frac{n}{2}}+\beta\right)}{8 \alpha^{2} n(n-1)^{2}}+\frac{(n-2)\left( \pm \varpi^{-\frac{n}{2}}+\beta\right)^{2}}{8 \alpha^{2} n^{2}(n-1)^{2}}\right)=0 .
$$

Integrating (3.35), we have

$$
\left(\frac{d \varpi}{d s}\right)^{2}+\varpi^{2}\left(1-\frac{\left( \pm \varpi^{-\frac{n}{2}}+\beta\right)^{2}}{4 \alpha^{2} n^{2}(n-1)^{2}}\right)=C,
$$

where $C$ is a constant. Thus, we have

$$
\varpi^{2}\left(1-\frac{\left( \pm \varpi^{-\frac{n}{2}}+\beta\right)^{2}}{4 \alpha^{2} n^{2}(n-1)^{2}}\right) \leq C .
$$

If the positive function $\varpi$ is not bounded, that is, $\lim _{s \rightarrow+\infty} \varpi(s)=+\infty$. From (3.37), we have

$$
+\infty\left(1-\frac{\beta^{2}}{4 \alpha^{2} n^{2}(n-1)^{2}}\right) \leq C
$$


Since $1-\frac{\beta^{2}}{4 \alpha^{2} n^{2}(n-1)^{2}}>0$, we have a contradiction from (3.38). Therefore, we know that $\varpi$ is bounded.

(2) Obviously, if $\lambda>0$, we know that $\varpi=e^{-\left(\lambda / \sigma \sqrt{\frac{n}{n-1}} \rho\right)}$ is bounded. This completes the proof of Lemma 3.2.

Proof of (2) in Theorem 1.1. Since $c=1$, if the sectional curvature of $M^{n}$ is nonnegative, that is, for $i \neq j, R_{i j i j}=1-\lambda_{i} \lambda_{j} \geq 0$, we have $1-\lambda \mu \geq 0$. From (3.33), we have $\frac{d^{2} \varpi}{d s^{2}} \leq 0$. Thus, $\frac{d \varpi}{d s}$ is a monotonic function of $s \in(-\infty,+\infty)$. Therefore, by the similar assertion in Wei [13], we have $\varpi(s)$ must be monotonic when $s$ tends to infinity. From the assumption of Theorem 1.1 and Lemma 3.2, we know that the positive function $\varpi(s)$ is bounded. Since $\varpi(s)$ is bounded and monotonic when $s$ tends to infinity, we know that both $\lim _{s \rightarrow-\infty} \varpi(s)$ and $\lim _{s \rightarrow+\infty} \varpi(s)$ exist and then we get

$$
\lim _{s \rightarrow-\infty} \frac{d \varpi(s)}{d s}=\lim _{s \rightarrow+\infty} \frac{d \varpi(s)}{d s}=0 .
$$

From the monotonicity of $\frac{d \varpi(s)}{d s}$, we have $\frac{d \varpi(s)}{d s} \equiv 0$ and $\varpi(s)=$ constant. Since we know that $\varpi=|2 \alpha n(n-1) \lambda-\beta|^{-\frac{2}{n}}$ for linear Weingarten spacelike hypersurface and $\varpi=e^{-\left(\lambda / \sigma \sqrt{\frac{n}{n-1}} \rho\right)}$ for constant $\rho$ Weingarten spacelike hypersurface, from (3.5) and (3.10), we have $\lambda$ and $\mu$ are constant, that is, $M^{n}$ is isoparametric. By the congruence Theorem of Abe, Koike and Yamaguchi (see Theorem 5.1 of [1]), we know that $M^{n}$ is isometric to the hyperbolic cylinder $H^{1}\left(1-\operatorname{coth}^{2} \varrho\right) \times S^{n-1}\left(1-\tanh ^{2} \varrho\right)$ or spherical cylinder $H^{n-1}\left(1-\operatorname{coth}^{2} \varrho\right) \times S^{1}\left(1-\tanh ^{2} \varrho\right)$. This completes the proof of $(2)$ in Theorem 1.1 .

We prove the following Lemmas:

Lemma 3.3. Let

$$
P(t)=1-\frac{\beta}{4 \alpha(n-1)} t+\frac{n-2}{2} t^{2},
$$

and $t^{\prime}=\frac{\beta}{4 \alpha(n-1)(n-2)}$. If $1-\frac{(n-2) \beta^{2}}{2 n^{4}(n-1) \alpha^{2}}<0$, then $P(t)$ has two distinct real roots $t_{1}, t_{2}$ and

(i) if $t \geq t^{\prime}$, then $t \geq t_{2}$ holds if and only if $P(t) \geq 0$ and $t \leq t_{2}$ holds if and only if $P(t) \leq 0$.

(ii) if $t \leq t^{\prime}$, then $t \leq t_{1}$ holds if and only if $P(t) \geq 0$ and $t \geq t_{1}$ holds if and only if $P(t) \leq 0$.

Proof. We have

$$
\frac{d P(t)}{d t}=-\frac{\beta}{4 \alpha(n-1)}+(n-2) t .
$$


it follows that the solution of $\frac{d P_{H}(t)}{d t}=0$ is $t^{\prime}=\frac{\beta}{4 \alpha(n-1)(n-2)}$. Therefore, we know that $t \geq t^{\prime}$ if and only if $P(t)$ is an increasing function, $t \leq t^{\prime}$ if and only if $P(t)$ is a decreasing function and $P(t)$ obtains its minimum at $t=t^{\prime}$.

Since $P(t)$ is continuous and $1-\frac{(n-2) \beta^{2}}{2 n^{4}(n-1) \alpha^{2}}<0$, we have $P\left(t^{\prime}\right)=1-$ $\frac{\beta^{2}}{32 \alpha^{2}(n-1)^{2}(n-2)}<0$. Therefore, we know that $P(t)$ has two distinct real roots $t_{1}, t_{2}$ and $t_{1}<t^{\prime}<t_{2}$.

(i) If $t \geq t^{\prime}$, from the increasing property of $P(t)$, we obtain that $t \geq t_{2}$ holds if and only if $P(t) \geq P\left(t_{2}\right)=0$ and $t \leq t_{2}$ holds if and only if $P(t) \leq$ $P\left(t_{2}\right)=0$.

(ii) If $t \leq t^{\prime}$, from the decreasing property of $P(t)$, we obtain that $t \leq t_{1}$ holds if and only if $P(t) \geq P\left(t_{1}\right)=0$ and $t \geq t_{1}$ holds if and only if $P(t) \leq P\left(t_{1}\right)=0$. This completes the proof of Lemma 3.3.

By the same method of the proof of Lemma 3.3, we may obtain

Lemma 3.4. Let

$$
\tilde{P}(t)=-t^{2}+\sigma \sqrt{\frac{n}{n-1}} \rho t+1,
$$

and $\tilde{t^{\prime}}=\frac{\sigma}{2} \sqrt{\frac{n}{n-1}} \rho$. Then $\tilde{P}(t)$ has two distinct real roots $\tilde{t_{1}}, \tilde{t_{2}}, \tilde{t_{1}}<\tilde{t^{\prime}}<\tilde{t_{2}}$ and

(i) if $t \geq \tilde{t^{\prime}}$, then $t \geq \tilde{t}_{2}$ holds if and only if $\tilde{P}(t) \leq 0$ and $t \leq \tilde{t}_{2}$ holds if and only if $\tilde{P}(t) \geq 0$.

(ii) if $t \leq \tilde{t^{\prime}}$, then $t \leq \tilde{t}_{1}$ holds if and only if $\tilde{P}(t) \leq 0$ and $t \geq \tilde{t}_{1}$ holds if and only if $\tilde{P}(t) \geq 0$.

From (3.5), we have the squared norm of the second fundamental form of $M^{n}$ is

$$
\begin{aligned}
S(t) & =(n-1) \lambda^{2}+\mu^{2} \\
& =\frac{n^{2}}{4} \lambda^{2}-\frac{(n-2) \beta}{4 \alpha(n-1)} \lambda+\frac{\beta^{2}}{16 \alpha^{2}(n-1)^{2}} .
\end{aligned}
$$

Putting $t=\lambda$, we have the following Lemma:

Lemma 3.5. Let

$$
S(t)=\frac{n^{2}}{4} t^{2}-\frac{(n-2) \beta}{4 \alpha(n-1)} t+\frac{\beta^{2}}{16 \alpha^{2}(n-1)^{2}},
$$

and $t^{\prime \prime}=\frac{(n-2) \beta}{2 \alpha n^{2}(n-1)}$. If $1-\frac{(n-2) \beta^{2}}{2 n^{4}(n-1) \alpha^{2}}<0$, then 
(i) If $t \geq t^{\prime \prime}$, then $t \geq t_{2}$ holds if and only if $S(t) \geq S\left(t_{2}\right)$ and $t \leq t_{2}$ holds if and only if $S(t) \leq S\left(t_{2}\right)$.

(ii) If $t \leq t^{\prime \prime}$, then $t \leq t_{1}$ holds if and only if $S(t) \geq S\left(t_{1}\right)$ and $t \geq t_{1}$ holds if and only if $S(t) \leq S\left(t_{1}\right)$.

Proof. We have

$$
\frac{d S(t)}{d t}=\frac{n^{2}}{2} t-\frac{(n-2) \beta}{4 \alpha(n-1)},
$$

it follows that the solution of $\frac{d S(t)}{d t}=0$ is $t^{\prime \prime}=\frac{(n-2) \beta}{2 \alpha n^{2}(n-1)}$. Therefore, we know that if $t \geq t^{\prime \prime}$ if and only if $S(t)$ is an increasing function, $t \leq t^{\prime \prime}$ if and only if $S(t)$ is a decreasing function and $S(t)$ obtain its minimum at $t^{\prime \prime}=\frac{(n-2) \beta}{2 \alpha n^{2}(n-1)}$.

Since $1-\frac{(n-2) \beta^{2}}{2 n^{4}(n-1) \alpha^{2}}<0$, we have $P\left(t^{\prime \prime}\right)=1-\frac{(n-2) \beta^{2}}{2 \alpha^{2} n^{4}(n-1)}<0$. Thus, we have $t_{1}<t^{\prime \prime}<t_{2}$.

(i) If $t \geq t^{\prime \prime}$, from the increasing property of $S(t)$, we obtain that $t \geq t_{2}$ holds if and only if $S(t) \geq S\left(t_{2}\right)$ and $t \leq t_{2}$ holds if and only if $S(t) \leq S\left(t_{2}\right)$.

(ii) If $t \leq t^{\prime \prime}$, from the decreasing property of $S(t)$, we obtain that $t \leq t_{1}$ holds if and only if $S(t) \geq S\left(t_{1}\right)$ and $t \geq t_{1}$ holds if and only if $S(t) \leq S\left(t_{1}\right)$. This completes the proof of Lemma 3.5.

From (3.10), we have the mean curvature of $M^{n}$ is

$$
H=\frac{n-1}{n} \lambda+\frac{1}{n} \mu=\lambda-\frac{\sigma}{n} \sqrt{\frac{n}{n-1}} \rho .
$$

Putting $t=\lambda$, we easily have:

Lemma 3.6. Let

$$
H(t)=t-\frac{\sigma}{n} \sqrt{\frac{n}{n-1}} \rho .
$$

Then $H(t)$ is an increasing function.

Proof of Theorem 1.2. (1) For linear Weingarten spacelike hypersurface, putting $t=\lambda$, from (3.34), we have

$$
\frac{d^{2} \varpi}{d s^{2}}+\varpi P(t)=0
$$

(i) If $\min \left(S\left(t_{1}\right), S\left(t_{2}\right)\right) \leq S(t) \leq \max \left(S\left(t_{1}\right), S\left(t_{2}\right)\right)$, then we have $S\left(t_{1}\right) \leq$ $S(t) \leq S\left(t_{2}\right)$ or $S\left(t_{2}\right) \leq S(t) \leq S\left(t_{1}\right)$.

(a) If $S\left(t_{1}\right) \leq S(t) \leq S\left(t_{2}\right)$, we consider two cases $t \geq t^{\prime \prime}$ or $t<t^{\prime \prime}$. case (i). If $t \geq t^{\prime \prime}$, we also consider two subcases $t^{\prime \prime} \geq t^{\prime}$ or $t^{\prime \prime}<t^{\prime}$. 
subcase (i). If $t^{\prime \prime} \geq t^{\prime}$, we have $t \geq t^{\prime}$. Since $S(t) \leq S\left(t_{2}\right)$, from Lemma 3.5, Lemma 3.3 and (3.45), we have $S(t) \leq S\left(t_{2}\right)$ holds if and only if $t \leq t_{2}$ if and only if $P(t) \leq 0$ and if and only if $\frac{d^{2} \varpi}{d s^{2}} \geq 0$. Thus $\frac{d \varpi}{d s}$ is a monotonic function of $s \in(-\infty,+\infty)$. Therefore, by the similar assertion in Wei [13], we have $\varpi(s)$ must be monotonic when $s$ tends to infinity. From the assumption of Theorem 1.2 and Lemma 3.2, we have the positive function $\varpi=|2 \alpha n(n-1) \lambda-\beta|^{-\frac{2}{n}}$ is bounded. By the same assertion in the proof of (2) in Theorem 1.1, we know that $M^{n}$ is isometric to the hyperbolic cylinder $H^{1}\left(1-\operatorname{coth}^{2} \varrho\right) \times S^{n-1}(1-$ $\left.\tanh ^{2} \varrho\right)$ or spherical cylinder $H^{n-1}\left(1-\operatorname{coth}^{2} \varrho\right) \times S^{1}\left(1-\tanh ^{2} \varrho\right)$.

subcase (ii). If $t^{\prime \prime}<t^{\prime}$, since $t \geq t^{\prime \prime}$, we have $t^{\prime \prime} \leq t<t^{\prime}$ or $t \geq t^{\prime}$.

If $t^{\prime \prime} \leq t<t^{\prime}$, from the decreasing property of $P(t)$, we have $P(t) \leq$ $P\left(t^{\prime \prime}\right)<0$. From (3.45), we have $\frac{d^{2} \varpi}{d s^{2}}>0$. This implies that $\frac{d \varpi(s)}{d s}$ is a strictly monotone increasing function of $s$ and thus it has at most one zero point for $s \in(-\infty,+\infty)$. If $\frac{d \varpi(s)}{d s}$ has no zero point in $(-\infty,+\infty)$, then $\varpi(s)$ is a monotone function of $s$ in $(-\infty,+\infty)$. If $\frac{d \varpi(s)}{d s}$ has exactly one zero point $s_{0}$ in $(-\infty,+\infty)$, then $\varpi(s)$ is a monotone function of $s$ in both $\left(-\infty, s_{0}\right]$ and $\left[s_{0},+\infty\right)$.

On the other hand, from Lemma 3.2, we know that $\varpi(s)$ is bounded. Since $\varpi(s)$ is bounded and monotonic when $s$ tends to infinity, we know that both $\lim _{s \rightarrow-\infty} \varpi(s)$ and $\lim _{s \rightarrow+\infty} \varpi(s)$ exist and (3.39) holds. This is impossible because $\frac{d \varpi(s)}{d s}$ is a strictly monotone increasing function of $s$. Therefore, we know that the case $t^{\prime \prime} \leq t<t^{\prime}$ does not occur and we conclude that $t \geq t^{\prime}$.

If $t \geq t^{\prime}$, then $t>t^{\prime \prime}$. Since $S(t) \leq S\left(t_{2}\right)$, from Lemma 3.5, Lemma 3.3 and (3.45), we have $S(t) \leq S\left(t_{2}\right)$ holds if and only if $t \leq t_{2}$ if and only if $P(t) \leq 0$ and if and only if $\frac{d^{2} \varpi}{d s^{2}} \geq 0$. Thus $\frac{d \varpi}{d s}$ is a monotonic function of $s \in(-\infty,+\infty)$. By the same assertion in the proof of (2) in Theorem 1.1, we know that $(i)$ in (1) of Theorem 1.2 is true.

case (ii). If $t<t^{\prime \prime}$, we also consider two subcases $t^{\prime \prime} \geq t^{\prime}$ or $t^{\prime \prime}<t^{\prime}$.

subcase (i). If $t^{\prime \prime} \geq t^{\prime}$, since $t<t^{\prime \prime}$, we have $t^{\prime}<t<t^{\prime \prime}$ or $t \leq t^{\prime}$.

If $t^{\prime}<t<t^{\prime \prime}$, from the increasing property of $P(t)$, we have $P(t)<$ $P\left(t^{\prime \prime}\right)<0$. From (3.45), we have $\frac{d^{2} \varpi}{d s^{2}}>0$. This implies that $\frac{d \varpi(s)}{d s}$ is a strictly monotone increasing function of $s$ and thus it has at most one zero point for $s \in(-\infty,+\infty)$. By the same assertion in the proof of case (i), we know that the case $t^{\prime}<t<t^{\prime \prime}$ does not occur and we conclude that $t \leq t^{\prime}$.

If $t \leq t^{\prime}$, since $t<t^{\prime \prime}$ and $S(t) \geq S\left(t_{1}\right)$, from Lemma 3.5, Lemma 3.3 and (3.45), we have $S(t) \geq S\left(t_{1}\right)$ holds if and only if $t \leq t_{1}$ if and only if $P(t) \geq 0$ and if and only if $\frac{d^{2} \varpi}{d s^{2}} \leq 0$. Thus $\frac{d \varpi}{d s}$ is a monotonic function of $s \in(-\infty,+\infty)$. By the same assertion in the proof of (2) in Theorem 1.1, we know that $(i)$ in (1) of Theorem 1.2 is true.

subcase (ii). If $t^{\prime \prime}<t^{\prime}$, since $t<t^{\prime \prime}$, we have $t<t^{\prime}$. Since $S(t) \geq S\left(t_{1}\right)$, 
from Lemma 3.5, Lemma 3.3 and (3.45), we have $S(t) \geq S\left(t_{1}\right)$ holds if and only if $t \leq t_{1}$ if and only if $P(t) \geq 0$ and if and only if $\frac{d^{2} \varpi}{d s^{2}} \leq 0$. Thus $\frac{d \varpi}{d s}$ is a monotonic function of $s \in(-\infty,+\infty)$. By the same assertion in the proof of (2) in Theorem 1.1, we know that $(i)$ in (1) of Theorem 1.2 is true.

(b) If $S\left(t_{2}\right) \leq S(t) \leq S\left(t_{1}\right)$, we also consider two cases $t \geq t^{\prime \prime}$ or $t<t^{\prime \prime}$. By the same assertion in the proof of $(a)$, we know that $(i)$ in (1) of Theorem 1.2 is true.

(ii) If $S(t) \geq \max \left(S\left(t_{1}\right), S\left(t_{2}\right)\right)$, we consider two cases $t \geq t^{\prime \prime}$ or $t<t^{\prime \prime}$. case (i). If $t \geq t^{\prime \prime}$, we also consider two subcases $t^{\prime \prime} \geq t^{\prime}$ or $t^{\prime \prime}<t^{\prime}$.

subcase (i). If $t^{\prime \prime} \geq t^{\prime}$, we have $t \geq t^{\prime}$. Since $S(t) \geq \max \left(S\left(t_{1}\right), S\left(t_{2}\right)\right)$, we have $S(t) \geq S\left(t_{2}\right)$, from Lemma 3.5, Lemma 3.3 and (3.45), we have $S(t) \geq S\left(t_{2}\right)$ holds if and only if $t \geq t_{2}$ if and only if $P(t) \geq 0$ and if and only if $\frac{d^{2} \varpi}{d s^{2}} \leq 0$. Thus $\frac{d \varpi}{d s}$ is a monotonic function of $s \in(-\infty,+\infty)$. By the same assertion in the proof of (2) in Theorem 1.1, we know that (ii) in (1) of Theorem 1.2 is true.

subcase (ii). If $t^{\prime \prime}<t^{\prime}$, since $t \geq t^{\prime \prime}$, we have $t^{\prime \prime} \leq t<t^{\prime}$ or $t \geq t^{\prime}$.

If $t^{\prime \prime} \leq t<t^{\prime}$, from the decreasing property of $P(t)$, we have $P(t)<$ $P\left(t^{\prime \prime}\right)<0$. From (3.45), we have $\frac{d^{2} \varpi}{d s^{2}}>0$. This implies that $\frac{d \varpi(s)}{d s}$ is a strictly monotone increasing function of $s$. By the same assertion in the proof of case (i) in (i), we know that the case $t^{\prime \prime} \leq t<t^{\prime}$ does not occur and we conclude that $t \geq t^{\prime}$.

If $t \geq t^{\prime}$, then $t>t^{\prime \prime}$. Since $S(t) \geq S\left(t_{2}\right)$, from Lemma 3.5, Lemma 3.3 and (3.45), we have $\frac{d^{2} \varpi}{d s^{2}} \leq 0$. By the same assertion above, we know that $(i i)$ in (1) of Theorem 1.2 is true.

case (ii). If $t<t^{\prime \prime}$, we also consider two subcases $t^{\prime \prime} \geq t^{\prime}$ or $t^{\prime \prime}<t^{\prime}$.

subcase (i). If $t^{\prime \prime} \geq t^{\prime}$, since $t<t^{\prime \prime}$, we have $t^{\prime}<t<t^{\prime \prime}$ or $t \leq t^{\prime}$.

If $t^{\prime}<t<t^{\prime \prime}$, from the increasing property of $P(t)$, we have $P(t)<$ $P\left(t^{\prime \prime}\right)<0$. From (3.45), we have $\frac{d^{2} \varpi}{d s^{2}}>0$. This implies that $\frac{d \varpi(s)}{d s}$ is a strictly monotone increasing function of $s$. By the same assertion in the proof of case (i) in (i), we know that the case $t^{\prime}<t<t^{\prime \prime}$ does not occur and we conclude that $t \leq t^{\prime}$.

If $t \leq t^{\prime}$, since $t<t^{\prime \prime}$ and $S(t) \geq \max \left(S\left(t_{1}\right), S\left(t_{2}\right)\right)$, we have $S(t) \geq S\left(t_{1}\right)$, from Lemma 3.5, Lemma 3.3 and (3.45), we have $S(t) \geq S\left(t_{1}\right)$ holds if and only if $t \leq t_{1}$ if and only if $P(t) \geq 0$ and if and only if $\frac{d^{2} \varpi}{d s^{2}} \leq 0$. Thus $\frac{d \varpi}{d s}$ is a monotonic function of $s \in(-\infty,+\infty)$. By the same assertion in the proof of (2) in Theorem 1.1, we know that (ii) in (1) of Theorem 1.2 is true.

subcase (ii). If $t^{\prime \prime}<t^{\prime}$, since $t<t^{\prime \prime}$, we have $t<t^{\prime}$. Since $S(t) \geq S\left(t_{1}\right)$, from Lemma 3.5, Lemma 3.3 and (3.45), we have $\frac{d^{2} \varpi}{d s^{2}} \leq 0$. By the same assertion above, we know that $(i i)$ in (1) of Theorem 1.2 is true.

(iii) If $S(t) \leq \min \left(S\left(t_{1}\right), S\left(t_{2}\right)\right)$, we consider two cases $t \geq t^{\prime \prime}$ or $t<t^{\prime \prime}$. 
case (i). If $t \geq t^{\prime \prime}$, we also consider two subcases $t^{\prime \prime} \geq t^{\prime}$ or $t^{\prime \prime}<t^{\prime}$. subcase (i). If $t^{\prime \prime} \geq t^{\prime}$, we have $t \geq t^{\prime}$. Since $S(t) \leq \min \left(S\left(t_{1}\right), S\left(t_{2}\right)\right)$, we have $S(t) \leq S\left(t_{2}\right)$, from Lemma 3.5, Lemma 3.3 and (3.45), we have $S(t) \leq S\left(t_{2}\right)$ holds if and only if $t \leq t_{2}$ if and only if $P(t) \leq 0$ and if and only if $\frac{d^{2} \varpi}{d s^{2}} \geq 0$. Thus $\frac{d \varpi}{d s}$ is a monotonic function of $s \in(-\infty,+\infty)$. By the same assertion in the proof of (2) in Theorem 1.1, we know that (iii) in (1) of Theorem 1.2 is true.

subcase (ii). If $t^{\prime \prime}<t^{\prime}$, since $t \geq t^{\prime \prime}$, we have $t^{\prime \prime} \leq t<t^{\prime}$ or $t \geq t^{\prime}$.

If $t^{\prime \prime} \leq t<t^{\prime}$, from the decreasing property of $P(t)$, we have $P(t) \leq$ $P\left(t^{\prime \prime}\right)<0$. From (3.45), we have $\frac{d^{2} \varpi}{d s^{2}}>0$. By the same assertion in the proof of case (i) in (i), we know that the case $t^{\prime \prime} \leq t<t^{\prime}$ does not occur and we conclude that $t \geq t^{\prime}$.

If $t \geq t^{\prime}$, then $t>t^{\prime \prime}$. Since $S(t) \leq S\left(t_{2}\right)$, from Lemma 3.5, Lemma 3.3 and (3.45), we have $\frac{d^{2} \varpi}{d s^{2}} \geq 0$. By the same assertion above, we also know that (iii) in (1) of Theorem 1.2 is true.

case (ii). If $t<t^{\prime \prime}$, we also consider two subcases $t^{\prime \prime} \geq t^{\prime}$ or $t^{\prime \prime}<t^{\prime}$. subcase (i). If $t^{\prime \prime} \geq t^{\prime}$, since $t<t^{\prime \prime}$, we have $t^{\prime}<t<t^{\prime \prime}$ or $t \leq t^{\prime}$.

If $t^{\prime}<t<t^{\prime \prime}$, from the increasing property of $P(t)$, we have $P(t)<P\left(t^{\prime \prime}\right)<$ 0 . From (3.45), we have $\frac{d^{2} \varpi}{d s^{2}}>0$. By the same assertion in the proof of case (i) in (i), we know that the case $t^{\prime}<t<t^{\prime \prime}$ does not occur and we conclude that $t \leq t^{\prime}$.

If $t \leq t^{\prime}$, since $t<t^{\prime \prime}$ and $S(t) \leq \min \left(S\left(t_{1}\right), S\left(t_{2}\right)\right)$, we have $S(t) \leq S\left(t_{1}\right)$, from Lemma 3.5, Lemma 3.3 and (3.45), we have $S(t) \leq S\left(t_{1}\right)$ holds if and only if $t \geq t_{1}$ if and only if $P(t) \leq 0$ and if and only if $\frac{d^{2} \varpi}{d s^{2}} \geq 0$. Thus $\frac{d \varpi}{d s}$ is a monotonic function of $s \in(-\infty,+\infty)$. By the same assertion in the proof of (2) in Theorem 1.1, we know that (iii) in (1) of Theorem 1.2 is true.

subcase (ii). If $t^{\prime \prime}<t^{\prime}$, since $t<t^{\prime \prime}$, we have $t<t^{\prime}$. Since $S(t) \leq S\left(t_{1}\right)$, from Lemma 3.5, Lemma 3.3 and (3.45), we have $\frac{d^{2} \varpi}{d s^{2}} \geq 0$. By the same assertion above, we know that (iii) in (1) of Theorem 1.2 is true.

(2) For constant $\rho$ Weingarten spacelike hypersurface, putting $t=\lambda$, from (3.10) and (3.33), we have for $c=1$

$$
\frac{d^{2} \varpi}{d s^{2}}+\varpi \tilde{P}(t)=0 .
$$

(i) If $H(t) \geq H\left(\tilde{t_{2}}\right)$, from Lemma 3.4 , we have $t \geq \tilde{t_{2}}>\tilde{t^{\prime}}$. From Lemma 3.6, Lemma 3.4 and $(3.46)$, we have $H(t) \geq H\left(\tilde{t_{2}}\right)$ holds if and only if $t \geq \tilde{t_{2}}$ if and only if $\tilde{P}(t) \leq 0$ and if and only if $\frac{d^{2} \varpi}{d s^{2}} \geq 0$. Thus $\frac{d \varpi}{d s}$ is a monotonic function of $s \in(-\infty,+\infty)$. Therefore, by the similar assertion in Wei [13], we have $\varpi(s)$ must be monotonic when $s$ tends to infinity. From Lemma 3.2, we have the positive function $\varpi(s)$ is bounded if $\lambda>0$. By the same assertion 
in the proof of (2) in Theorem 1.1, we know that $M^{n}$ is isometric to the hyperbolic cylinder $H^{1}\left(1-\operatorname{coth}^{2} \varrho\right) \times S^{n-1}\left(1-\tanh ^{2} \varrho\right)$ or spherical cylinder $H^{n-1}\left(1-\operatorname{coth}^{2} \varrho\right) \times S^{1}\left(1-\tanh ^{2} \varrho\right)$.

(ii) If $H(t) \leq H\left(\tilde{t_{1}}\right)$, we have $t \leq \tilde{t_{1}}<\tilde{t^{\prime}}$. From Lemma 3.6, Lemma 3.4 and (3.46), we have $H(t) \leq H\left(\tilde{t_{1}}\right)$ holds if and only if $t \leq \tilde{t_{1}}$ if and only if $\tilde{P}(t) \leq 0$ and if and only if $\frac{d^{2} \varpi}{d s^{2}} \geq 0$. Thus $\frac{d \varpi}{d s}$ is a monotonic function of $s \in(-\infty,+\infty)$. By the same assertion above, we know that (ii) in (2) of Theorem 1.2 is true.

(iii) If $H\left(\tilde{t_{1}}\right) \leq H(t) \leq H\left(\tilde{t_{2}}\right)$, we have $\tilde{t_{1}} \leq t \leq \tilde{t_{2}}$. Since $\tilde{t_{1}} \leq \tilde{t^{\prime}} \leq \tilde{t_{2}}$, we consider two cases $t \geq \tilde{t^{\prime}}$ or $t<\tilde{t^{\prime}}$.

If $t \geq \tilde{t^{\prime}}$, since $H(t) \leq H\left(\tilde{t_{2}}\right)$, from Lemma 3.6, Lemma 3.4 and (3.46), we have $H(t) \leq H\left(\tilde{t_{2}}\right)$ holds if and only if $t \leq \tilde{t_{2}}$ if and only if $\tilde{P}(t) \geq 0$ and if and only if $\frac{d^{2} \varpi}{d s^{2}} \leq 0$. Thus $\frac{d \varpi}{d s}$ is a monotonic function of $s \in(-\infty,+\infty)$. By the same assertion above, we know that (iii) in (2) of Theorem 1.2 is true.

If $t<\tilde{t^{\prime}}$, since $H\left(\tilde{t_{1}}\right) \leq H(t)$, from Lemma 3.6, Lemma 3.4 and (3.46), we have $H\left(\tilde{t_{1}}\right) \leq H(t)$ holds if and only if $\tilde{t_{1}} \leq t$ if and only if $\tilde{P}(t) \geq 0$ and if and only if $\frac{d^{2} \varpi}{d s^{2}} \leq 0$. Thus $\frac{d \varpi}{d s}$ is a monotonic function of $s \in(-\infty,+\infty)$. By the same assertion above, we know that (iii) in (2) of Theorem 1.2 is true. This completes the proof of Theorem 1.2.

Acknowledgment. The authors would like to thank the referee for his many valuable suggestions and comments that really improve the paper.

Project supported by NSF of Shaanxi Educational Department(11JK0479).

\section{References}

[1] N. Abe, N. Koike and S. Yamaguchi, Congruence theorems for proper semi-Riemannian hypersurfaces in a real space form, Yokohama Math. J., 35(1987), no. 1-2, 123-136.

[2] K. Akutagawa, On spacelike hypersurfaces with constant mean curvature in a de Sitter space, Math. Z., 196(1987), no. 1, 13-19.

[3] J.A. Aledo, J.M. Espinar, A conformal representation for linear Weingarten surfaces in the de Sitter space, J. Geom. Phys., 57(2007), no. 8, 1669-1677.

[4] A.C. Asperti, R.M.B. Chaves and B.C. Valério, Ruled Weingarten hypersurfaces in the Lorentz-Minkowski space and in de Sitter space, J. Geom. Phys., 60(2010), no. 4, 553-561. 
[5] Z. Hu, M.Scherfner and S. Zhai, On spacelike hypersurfaces with constant scalar curvature in the de Sitter space, Differential Geom. Appl., 25(2007), no. $6,594-611$.

[6] C. Gerhardt, Curvature estimates for Weingarten hypersurfaces in Riemannian manifolds, Adv. Calc. Var., 1(2008), no. 1, 123-132.

[7] A. Brasil Jr., A. G. Colares and O. Palmas, Complete spacelike hypersurfaces with constant mean curvature in the de Sitter space: a gap Theorem, Illinois J. Math., 47(2003), no. 3, 847-866.

[8] H. Li. Global rigidity theorems of hypersurfaces, Ark. Mat., 35(1997), no. 2, 327-351.

[9] Z.H. Hou, D. Yang, Linear Weingarten spacelike hypersurfaces in de Sitter space, Bull. Belg. Math. Soc. Simon Stevin, 17(2010), no. 5, 769-780.

[10] O.C. Schnürer, The Dirichlet problem for Weingarten hypersurfaces in Lorentz manifolds, Math. Z., 242(2002), no. 1, 159-181.

[11] T. Otsuki, Minimal hypersurfaces in a Riemannian manifold of constant curvature, Amer. J. Math., 92(1970), no. 1, 145-173.

[12] S. Shu, Complete spacelike hypersurfaces in a de Sitter space, Bull. Austral Math. Soc., 73(2006), no. 1, 9-16.

[13] G. Wei, Complete hypersurfaces with constant mean curvature in a unit sphere, Monatsh. Math., 149(2006), no. 3, 251-258.

Junfeng Chen,

School of Mathematics and Information Science,

Xianyang Normal University,

Xianyang 712000 Shaanxi P.R. China.

Email: mailjunfeng@163.com

Shichang Shu,

School of Mathematics and Information Science,

Xianyang Normal University,

Xianyang 712000 Shaanxi P.R. China.

Email: shushichang@126.com 